\title{
Kikuchi-Fujimoto disease with multiple extra-nodal features - A clinical mimic
}

\author{
C.A. Mansoor ${ }^{1}$, Z. Shemin ${ }^{2}$ \\ 'Department of Internal Medicine; '2Department of pathology M.E.S. Medical College, \\ Perinthalmanna, Kerala, India
}

\begin{abstract}
SUMMARY
Extranodal involvement in Kikuchi's disease is uncommon. A 31-year-old previously healthy Indian woman was admitted with high grade fever, multiple joint pain and skin rash for 3 weeks. She had negative anti-nuclear antibodies and had features of Kikuchi's disease on lymph node biopsy. She also had multiple extranodal manifestations including erythematous maculopapular rash, symmetric polyarthritis and hepatosplenomegaly. Kikuchi's disease with extranodal involvement can clinically mimic diseases like hematological malignancies, connective tissue disorders and certain infections. A lymph node biopsy plays a crucial role in making an accurate diagnosis by excluding other diseases. A discussion on the importance of differentiating Kikuchi's disease from systemic lupus erythematosus is included.
\end{abstract}

Key words: Kikuchi's disease; Extra nodal features; clinical mimic.

Reumatismo, 2019; 71 (2): 105-107

\section{INTRODUCTION}

ikuchi's disease (KD) is an uncommon, benign, self-limited condition of unknown etiology which mainly affects young adults with a higher prevalence among Asians. It is clinically characterized by tender regional lymphadenopathy, fever, and occasional systemic involvement. Lymphomas, systemic lupus erythematosus (SLE), and certain infections have similar clinical and histopathological features, which makes it important to exclude such conditions to make a proper diagnosis of $\mathrm{KD}$.

\section{CASE REPORT}

A 31-year-old previously healthy Indian woman was admitted with high grade fever, multiple joint pain and skin rash for 3 weeks. She had pain involving large and small joints of upper and lower limbs with swelling and redness. She denied history of weight loss, had no sick contacts and had no history of addictions. She had no increased frequency of micturition, dysuria, loin pain, vaginal or urethral discharge, bleeding manifestations or mucosal ulcers.
Examination showed palpable posterior cervical lymph nodes which were firm in consistency, mobile, non-tender, and not matted. She had erythematous, non-scaly maculopapular rash over bilateral cheeks, forehead, anterior aspect of neck, central chest and extensor aspect of bilateral upper limbs.

Hemoglobin was $10.6 \mathrm{~g} / \mathrm{dL}(12-15 \mathrm{~g} / \mathrm{dL})$, erythrocyte sedimentation rate $65 \mathrm{~mm}$ in $1 \mathrm{~h}$ (normally less than $20 \mathrm{~mm} / \mathrm{hr}$ ) and C-reactive protein was $16 \mathrm{mg} / \mathrm{L}$ (normal range $0.8 \mathrm{mg} / \mathrm{L}$ to $3.0 \mathrm{mg} / \mathrm{L}$ ). Peripheral smear was normal. Urinalysis was normal. Biochemical parameters were normal. Chest radiography and electrocardiogram were normal. Etiological workup including Dengue, Leptospira, Brucella, Rickettsia, Epstein Barr virus, HIV, Hepatitis B and $\mathrm{C}$ serologies and antinuclear antibody was negative. The test for malarial parasite was negative and blood cultures did not reveal any growth. C3 and $\mathrm{C} 4$ levels were normal. Serum ferritin was $5000 \mathrm{microg} / \mathrm{L}$ $(10-200 \mathrm{mcg} / \mathrm{L})$. Ultrasonography of abdomen showed mild hepatosplenomegaly. Lymph node biopsy showed lymphoid follicle with germinal center, paracortical
Corresponding author: C. Abdulla Mansoor Department of General Medicine. M.E.S. Medical College, Perinthalmanna, Kerala 679338, India E-mail: drcamans@gmail.com 


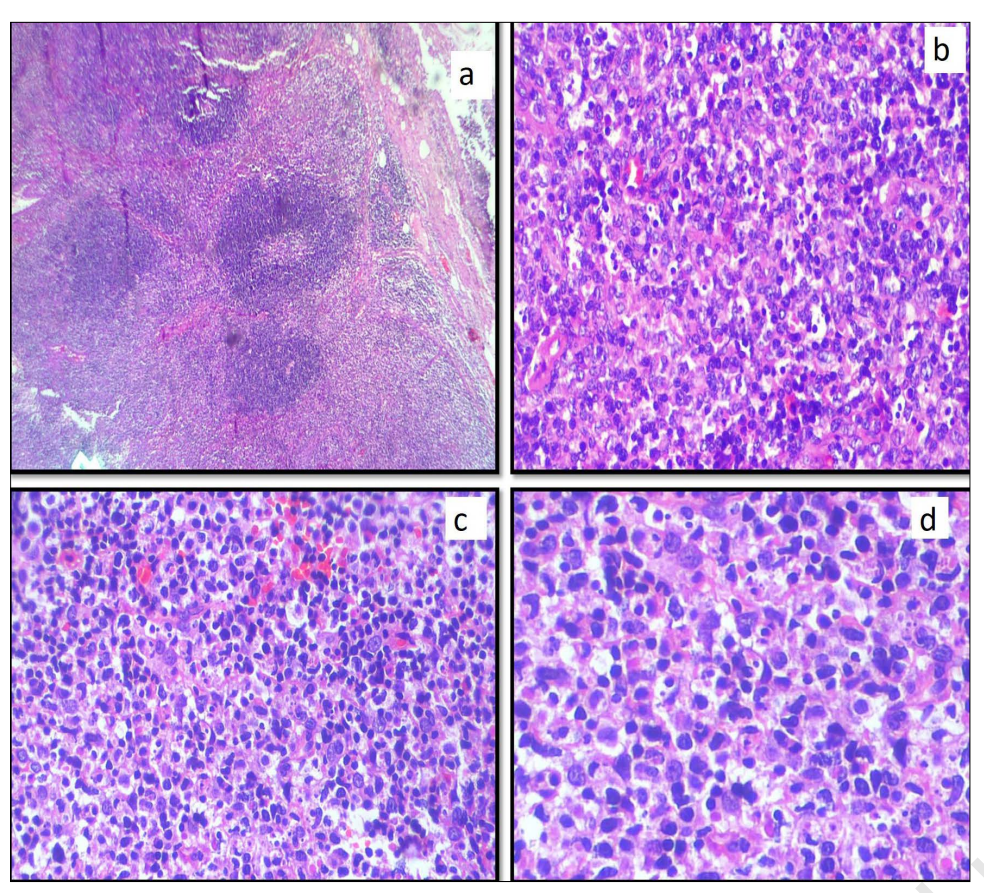

Figure 1 - Lymph node biopsy showing partially preserved architecture of lymphoid follicle with germinal center and paracortical area expansion (A), paracortical area expanded by histiocytes and many apoptotic bodies (B), crescent shaped nuclei of histiocytes (C), and patchy areas of cortical necrosis (D).

area expansion with histiocytes and many apoptotic bodies, crescentic histiocytes and patchy areas of cortical necrosis without neutrophils (Figure 1). Immunohistochemistry showed CD68 and Myeloperoxidase positive histiocytes characteristically seen in $\mathrm{KD}$ (Figure 2). A diagnosis of KD was made based on the investigations. She was started on oral prednisolone $1 \mathrm{mg} / \mathrm{kg}$, which was tapered and discontinued slowly over the next 8 weeks. When reviewed after 8 weeks she was afebrile, had no joint pain, skin rash or lymphoadenopathy, and serum ferritin was normal. She was followed up for one year and did not have any further complications.

\section{DISCUSSION AND CONCLUSIONS}

$\mathrm{KD}$ is a rare lymphohistiocytic disorder that affects young women of Asian descent more frequently than other ethnic groups. It is an enigmatic, benign, and self-limited syndrome characterized by regional lymphadenopathy and mild fever. Differential diagnosis of fever with lymphadenopathy and skin rash include infections, lymphoma and connective tissue diseases such as SLE. Extranodal involvement in KD is uncommon. Involvement of skin, bone marrow, nervous system, eye and liver dysfunction have been reported $(1,2)$. Dermatological manifestations reported previously with KD include; erythematous rashes and nodules, crusted papules; scattered, indurated, erythematous lesions; erythema multiforme; and erythematous maculopapular eruptions, predominantly involving the face and upper body (3). Generalized papulopustular skin lesions and lip edema with desquamation and erosions were also reported previously $(4,5)$. Hepatosplenomegaly may be associated with KD (6). KD with small and large joint polyarthritis has been described previously (7).

Our patient had KD with extra nodal mani-
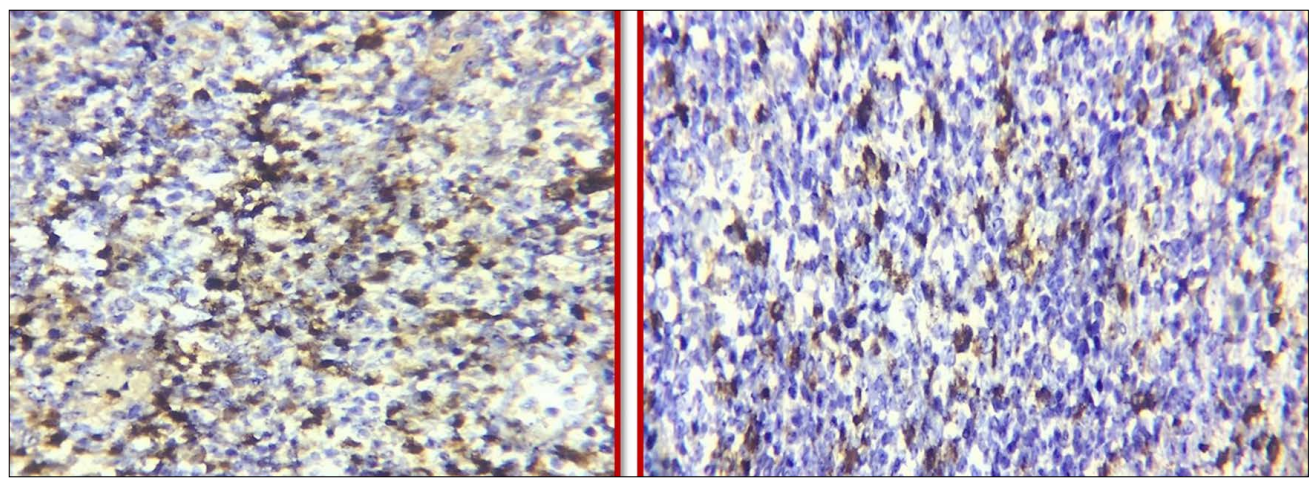

Figure 2 - Immunohistochemistry showing CD68 (A), and myeloperoxidase positive histiocytes (B). 
festations including erythematous maculopapular rash, symmetric polyarthritis and hepatosplenomegaly. The presentation of $\mathrm{KD}$ with predominant extranodal features makes it difficult for the physician to differentiate it from other disorders such as connective tissue disorders, especially SLE, lymphomas and certain infections. Lymph node biopsy is necessary to make an accurate diagnosis excluding other clinical mimics. SLE has been seen to either coincide, precede, or follow a diagnosis of KD. Among 35 cases of SLE-associated KD reviewed by Santana et al., seven patients were diagnosed with SLE prior to KD diagnosis, fourteen patients were diagnosed with KD and SLE simultaneously, and fourteen patients were diagnosed with SLE after a diagnosis of KD (8). Our patient did not have SLE during the diagnosis of KD or the follow-up period of one year. Lymph node biopsy and immunohistiochemistry in our patient showed classic features of KD without features of SLE (increased neutrophils, eosinophils, or plasma cells, and vasculitis and hematoxylin bodies).

This case is important for the following reasons. Firstly, young Asian women presenting with clinical features in keeping with a diagnosis of systemic lupus erythematosus or lymphoma should have a lymph node biopsy in order to rule out more benign conditions such as KD. Secondly, KD has been described in association with multiple extranodal manifestations including erythematous maculopapular rash, symmetric polyarthritis and hepatosplenomegaly.

\section{REFERENCES}

1. Yen A, Fearneyhough P, Raimer SS, et al. EBVassociated Kikuchi's histiocytic necrotizing lymphadenitis with cutaneous manifestations. J Am Acad Dermatol. 1997; 36: 342-36.

2. Yasukawa K, Matsumura T, Sato-Matsumura $\mathrm{KC}$, et al. Kikuchi's disease and the skin: case report and review of the literature. Br J Dermatol. 2001; 144: 885-9.

3. Kaur S, Thami GP, Mohan H, et al. Kikuchi disease with facial rash and erythema multiforme. Pediatr Dermatol. 2001; 18: 403-5.

4. Kim KJ, Jee MS, Chang SE, et al. Kikuchi-Fujimoto disease with papulopustular skin manifestations. Clin Exp Dermatol. 2003; 28: 142-4.

5. Imai K, Yokozeki H, Nishioka K. Kikuchi's disease (histiocytic necrotizing lymphadenitis) with cutaneous involvement. J Dermatol. 2002; 29: 587-92.

6. Al-Bishri J. Kikuchi Fujimoto Disease. Clin Med Insights Arthritis Musculoskelet Disord. 2012; 5: 63-6.

7. Graham LE. Kikuchi-Fujimoto disease and peripheral arthritis: a first!. Ann Rheum Dis. 2002; 61: 475.

8. Santana A, Lessa B, Galrão L, et al. KikuchiFujimoto's disease associated with systemic lupus erythematosus: case report and review of the literature. Clin Rheumatol. 2005; 24 : 60-3. 